This item was submitted to Loughborough's Research Repository by the author.

Items in Figshare are protected by copyright, with all rights reserved, unless otherwise indicated.

\title{
A note on the solution of regular PMDs
}

PLEASE CITE THE PUBLISHED VERSION

LICENCE

CC BY-NC-ND 4.0

\section{REPOSITORY RECORD}

Tan, L., and Ashley Clive Pugh. 2019. "A Note on the Solution of Regular Pmds". figshare. https://hdl.handle.net/2134/813. 


\title{
A note on the solution of regular PMDs
}

\author{
LIANSHENG TAN, A.C. PUGH \\ Department of Mathematical Sciences, Loughborough University \\ Loughborough, Leicestershire, LE11 3TU, UK
}

\begin{abstract}
A new resolvent decomposition of a regular polynomial matrix is derived which is a refinement of the results given by Gohberg et al (1982) and Vardulakis (1991). Based on this, a complete solution of the regular Polynomial Matrix Descriptions (PMDs) is presented that reflects precisely the zero input response and the zero state response, and takes into account the impulsive properties of the system. An algorithm for the computation of this resolvent decomposition is provided.
\end{abstract}

Corresponding Author:

Dr. A. C. Pugh

Department of Mathematical Sciences

Loughborough University

Leicestershire, LE11 3TU, UK

Telephone:

+44(0)1509223190

Telefax:

+44 (0)1509211869

E-mail:

A.C.Pugh@lboro.ac.uk 


\section{Introduction}

Regular Polynomial Matrix Descriptions (PMDs) are described by

$$
\Sigma^{\prime}:\left\{\begin{aligned}
T(\rho) \beta(t) & =E(\rho) u(t) \\
y(t) & =C(\rho) \beta(t)+D(\rho) u(t), \quad t \geq 0
\end{aligned}\right.
$$

where $\rho:=d / d t$ the differential operator, $T(\rho) \in R[\rho]^{l \times l}, \operatorname{rank}_{\Re[\rho]} T(\rho)=l, E(\rho) \in$ $R[\rho]^{l \times m^{\prime}}, C(\rho) \in R[\rho]^{p \times l}, D(\rho) \in R[\rho]^{p \times m^{\prime}}, \quad \beta(t):[0,+\infty) \rightarrow R^{l}$ is the pseudo state of $\Sigma^{\prime}, u(t):[0,+\infty) \rightarrow R^{m^{\prime}}$ is the control input and $y(t)$ the output of $\Sigma^{\prime}$. $\Sigma^{\prime}$ can be written in the form:

$$
\begin{gathered}
\underbrace{\left[\begin{array}{ccc}
T(\rho) & E(\rho) & 0 \\
-C(\rho) & D(\rho) & I_{p} \\
0 & -I_{m^{\prime}} & 0
\end{array}\right]}_{A(\rho)} \underbrace{\left[\begin{array}{c}
\beta(t) \\
-u(t) \\
y(t)
\end{array}\right]}_{\xi(t)}=\underbrace{\left[\begin{array}{c}
0 \\
0 \\
I_{m^{\prime}}
\end{array}\right]}_{B} u(t) \\
y(t)=\underbrace{\left[0,0, I_{p}\right]}_{\Psi} \underbrace{\left[\begin{array}{c}
\beta(t) \\
-u(t) \\
y(t)
\end{array}\right]}_{\xi(t)}, \quad t \geq 0 .
\end{gathered}
$$

In order to investigate the solution of the above PMD, we thus focus our interests on the following form of equation

$$
\Sigma: \quad A(\rho) \xi(t)=B u(t), \quad t \geq 0
$$

where $A(\rho) \in R[\rho]^{r \times r}$, with $\operatorname{rank}_{\Re[\rho]} A(\rho)=r, \xi(t) \in R^{r}, B \in R^{r \times m}, u(t) \in R^{m}$. The homogeneous case of $\Sigma$ is

$$
\Sigma_{0}: \quad A(\rho) \xi(t)=0, \quad t \geq 0 .
$$

Both regular generalized state space systems which are described by

$$
E \dot{x}=A x(t)+B u(t),
$$

where $E$ is a singular matrix, $\operatorname{rank}(\rho E-A)=r$, and the (regular) state space systems which are described by

$$
\dot{x}=A x(t)+B u(t)
$$

are special cases of the regular PMDs (1).

The solution of the above PMDs can be proposed on the basis of any resolvent decomposition of $A(s)$ given by

$$
A^{-1}(s)=C\left(s I_{n}-J\right)^{-1} Z+C_{\infty}\left(s J_{\infty}-I_{\varepsilon}\right)^{-1} Z_{\infty}
$$

where $(C, J)$ is the finite Jordan pair of $A(s)$, and $\left(C_{\infty}, J_{\infty}\right)$ is the infinite Jordan pair (Gohberg et al, 1982; Vardulakis, 1991) of $A(s)$. Such resolvent decompositions are not unique, but play an important role in formulating the solution of the regular PMDs. The difficulties in the problem of obtaining the solution of the PMD are specific to the 
particular resolvent decomposition used. From a computation point of view, when the matrices in (3) are of minimal dimensions, it is obviously easiest to obtain the inverse matrix of $A(s)$.

There is fundamental interest (notably Gohberg et al., 1982; Vardulakis, 1991) in the solution of the regular PMD and its homogeneous case. For (1) Gohberg et al. (1982) proposed a particular resolvent decomposition, and the solution of it was formulated according to this decomposition. However, the impulsive property of the system at $t=0$ was not properly considered in this work. One advantage of this resolvent decomposition however is that it is constructive, due to the fact that the matrices $Z, Z_{\infty}$ in (3) are formulated in terms of the finite and infinite Jordan pairs. On the other hand in this resolvent decomposition, there appears certain redundant information, in that the dimensions of the infinite Jordan pair are much larger than is usually necessary, this in turn bring some inconvenience in computing the inverse matrix of $A(s)$. In fact some of the infinite elementary divisors (Hayton, et al., 1988) which correspond to the infinite poles of $A(s)$ and actually contribute nothing to the solution. Thus the resolvent decomposition can be proposed in a simpler form which has the advantage of giving a more precise insight into the system structure and bringing some convenience in actual computation. Vardulakis (1991) obtained a general solution of (1) under the assumption that the initial conditions of $\xi(t)$ are zero. The main idea of this approach is to find the minimal realizations of the strictly proper part and the polynomial part of $A^{-1}(s)$ and then to obtain the required resolvent decomposition. Although this procedure does give good insight into the system structure, the realization approach is not so straightforward by itself and it is consequently more difficult to be applied in actual computation. Furthermore, in this procedure, no explicit formula for $Z$ and $Z_{\infty}$ is available. Apparently, differences arise between the solution of Gohberg et al (1982) and that of Vardulakis (1991) due to the fact that these two solutions are expressed through two different resolvent decompositions. Although it is found that the redundant information contained in the solution of Gohberg et al (1982) can be decoupled, an overly large resolvent decomposition definitely brings some inconvenience to actual computation.

The main purpose of this paper is to present a resolvent decomposition which is a refinement of both results obtained by Gohberg et al (1982) and Vardulakis (1991). It is formulated in terms of the notions of the finite Jordan pairs, infinite Jordan pairs and the generalised infinite Jordan pairs which were defined by Gohberg et al (1982) and Vardulakis (1991). We make clear the issue of infinite Jordan pair noted by Gohberg et al (1982) and Vardulakis (1991). This refined resolvent decomposition captures the essential feature of the system structure, the redundant information which is included in the resolvent decomposition of Gohberg et al (1982) is deleted through certain transformation, thus the resulting resolvent decomposition inherits the advantages of both results of Gohberg et al (1982) and that of Vardulakis (1991). In the proposed approach the matrices $Z, Z_{\infty}$ in (3) are formulated explicitly, which means this method is more constructive. The main idea in this proposed approach is to calculate an elementary matrix $P$, which is very easy to obtain, to delete the redundant information, then to propose the refined resolvent decomposition. This elementary matrix has the effect of deleting the redundant information in two ways. First, it deletes the redundant information in those blocks in the infinite Jordan pair of Gohberg et al (1982) which corresponds to the infinite zeros and bring them into the correct sizes. Second, it deletes the whole blocks in the infinite Jordan pair of Gohberg et al (1982) which corresponds to the infinite poles and the whole 
blocks which are not dynamically important. This elementary matrix serves to transform the partitioned block matrix in $Z_{\infty}$ which corresponds to the redundant information into zero, the resulting refined resolvent decomposition is thus of minimal dimensions. As well, by using this elementary matrix the mechanism of decoupling in the solution of Gohberg et al (1982) is explained clearly. This refined resolvent decomposition facilitates computation of the inverse matrix of $A(s)$ due to that the dimensions of the matrices used are of minimal. Rather than to calculate the inverse matrix $A^{-1}(s)$ with an overly large dimension, it is suggested to calculate the easily available elementary matrix $P$ at first to delete all the redundant information and then to get the inverse matrix with the minimal dimension. Once the refined resolvent decomposition is obtained, the generalized infinite Jordan pair and the elementary matrix $P$ are not needed to be involved in calculation of the solution of the regular PMDs. This thus presents another merit of this method which is algorithmically attractive when applied in actual computation.

Based on this proposed resolvent decomposition, a complete solution of a PMD follows which reflects the detailed structure of the zero state response and the zero input response of the system. The complete impulsive properties of the system are also dis-

played in our solution. Such impulsive properties is not properly displayed in the solution of Gohberg et al (1982). Although for the case of homogeneous case it is considered in Vardulakis (1991), such a complete impulsive properties of the system for the general nonhomogeneous regular PMDs is not available from Vardulakis (1991).

\section{Infinite Jordan Pairs}

In this section, we analyse two definitions of infinite Jordan pairs. The first was given by Gohberg et al. (1982) and the other was given by Vardulakis (1991). The essential differences between them are elucidated and these discussions enable us to establish a refined resolvent decomposition.

Let $A(s)=A_{0}+A_{1} s+\cdots+A_{q_{1}} s^{q_{1}} \in R^{r \times r}[s], \operatorname{rank}_{\Re[s]} A(s)=r . S_{A(s)}^{\infty}$ denotes the Smith-McMillan form of $A(s)$ at $s=\infty$,

$$
S_{A(s)}^{\infty}(s)=\operatorname{diag}[\underbrace{s^{q_{1}}, s^{q_{2}}, \cdots, s^{q_{k}}}_{k}, \underbrace{1, \cdots, 1}_{r-h-k}, \underbrace{1 / s^{\hat{q}_{r-h+1}}, \cdots, 1 / s^{\hat{q}_{r}}}_{h}]
$$

where

$$
q_{1} \geq q_{2} \geq \cdots \geq q_{k}>0
$$

are the orders of the infinite poles at $s=\infty$ of $A(s)$, and

$$
\hat{q}_{r} \geq \hat{q}_{r-1} \geq \cdots \geq \hat{q}_{r-h+1}>0
$$

are the orders of the infinite zeros at $s=\infty$ of $A(s)$. The dual polynomial of $A(s)$ is defined as

$$
D_{A(s)}(w):=w^{q_{1}} A\left(\frac{1}{w}\right)=A_{0} w^{q_{1}}+A_{1} w^{q_{1}-1}+\cdots+A_{q_{1}} .
$$

The local Smith form of $D_{A(s)}(w)$ at $w=0$ is $S_{D_{A(s)}}^{0}(w)=w^{q_{1}} S_{A(s)}^{\infty}\left(\frac{1}{w}\right)=\operatorname{diag}\left[1, w^{q_{1}-q_{2}}, \cdots, w^{q_{1}-q_{k}}, w^{q_{1}}, \cdots, w^{q_{1}}, w^{q_{1}+\hat{q}_{r-h+1}}, \cdots, w^{q_{1}+\hat{q}_{r}}\right]$. 
It is seen that the polynomial matrices have in general three kinds of IEDs, or equivalently $D_{A(s)}(w)$ has three kinds of finite zero at $w=0$. The first kind of IEDs with orders $q_{1}-q_{j}$, $j=2,3, \cdots, k$ correspond to the poles of $A(s)$ at $s=\infty$. The second kind of IEDs are those corresponding to the zeros of $A(s)$ at $s=\infty$ with orders $q_{1}+\hat{q}_{j}, j=r-h+1$. $\cdots, r$. The third kind with orders $q_{1}$ are not dynamically important (Hayton et al, 1988).

Let $\tilde{U}_{L}(w) \in R[s]^{r \times r}, \tilde{U}_{R}(w) \in R[s]^{r \times r}$ be unimodular matrices reducing $D_{A(s)}(w)$ to its local Smith form $S_{D_{A(s)}}^{0}(w)$, i.e.,

$$
\tilde{U}_{L}(w) D_{A(s)}(w) \tilde{U}_{R}(w)=S_{D_{A(s)}}^{0}(w) .
$$

Let $u_{j}(w) \in R[w]^{r \times 1}, v_{j}(w) \in R[w]^{r \times 1}, j=1,2, \cdots, r$ be the columns of $\tilde{U}_{R}(w)$ and $\tilde{U}_{L}^{-1}(w)$, then

$$
D_{A(s)}(w) u_{j}(w)=v_{j}(w) w^{\tau(j)}
$$

where

$$
\tau(j):= \begin{cases}q_{1}-q_{j} & , \quad j=1,2, \cdots, k \\ q_{1}+\hat{q}_{j} & , \quad j=k+1, \cdots, r\end{cases}
$$

Let

$$
\beta_{j q}:=\frac{1}{q !} u_{j}^{(q)}(0), \quad j=2,3, \cdots, r ; \quad q=0,1,2, \cdots, \tau(j)-1,
$$

then for $j=2,3, \cdots, r$, the vectors

$$
\beta_{j 0}, \beta_{j 1}, \cdots, \beta_{j(\tau(j)-1)} \in R^{r}
$$

form a Jordan chain corresponding to the zero $w=0$ of the IEDs $w^{\tau(j)}$. Now we can compare the two different definitions of the infinite Jordan pair.

Definition 1 (Gohberg et al, 1982): The generalised infinite Jordan pair $\left(C_{\infty}^{\prime}, J_{\infty}^{\prime}\right)$ of $A(s)$ are defined as

$$
\begin{gathered}
C_{\infty}^{\prime}:=\left[C_{2}^{\prime}, C_{3}^{\prime}, \cdots, C_{k}^{\prime}, C_{k+1}^{\prime}, \cdots, C_{r}^{\prime}\right] \in R^{r \times \nu}, \\
J_{\infty}^{\prime}:=\operatorname{block} \operatorname{diag}\left[J_{2}^{\prime}, \cdots, J_{r}^{\prime}\right] \in R^{\nu \times \nu}
\end{gathered}
$$

where

$$
\begin{aligned}
C_{j}^{\prime} & =\left[\beta_{j 0}, \beta_{j 1}, \cdots, \beta_{j(\tau(j)-1))}\right] \in R^{r \times \tau(j)}, \quad j=2,3, \cdot, r, \\
J_{j}^{\prime} & =\left[\begin{array}{ccccc}
0 & 1 & 0 & \cdots & 0 \\
0 & 0 & 1 & \cdots & 0 \\
\cdot & \cdot & \cdot & \cdots & \cdot \\
0 & 0 & 0 & \cdots & 1 \\
0 & 0 & 0 & \cdots & 0
\end{array}\right] \in R^{\tau(j) \times \tau(j)}, \quad j=2, \cdots, r .
\end{aligned}
$$

We observe that

1. $J_{\infty}^{\prime}$ is nilpotent with index of nilpotency $q_{1}+\hat{q}_{r}$, because the largest Jordan block is $\left(q_{1}+\hat{q}_{r}\right) \times\left(q_{1}+\hat{q}_{r}\right)$. 
2. $\nu=r q_{1}-\sum_{i=1}^{k} q_{i}$ (the total number of the infinite poles of $\mathrm{A}(\mathrm{s})$ )

$+\sum_{i=1}^{r} \hat{q}_{k+i} \quad$ (the total number of the infinite zeros of $\mathrm{A}(\mathrm{s})$ )

Definition 2 (Vardulakis, 1991): The infinite Jordan pair $\left(C_{\infty}, J_{\infty}\right)$ of $A(s)$ is defined as follows

$$
\begin{gathered}
C_{\infty}:=\left[C_{k+1}, \cdots, C_{r}\right] \in R^{r \times \mu}, \\
J_{\infty}:=\text { blockdiag }\left[J_{k+1}, \cdots, J_{r}\right] \in R^{\mu \times \mu},
\end{gathered}
$$

where

$$
\begin{gathered}
C_{j}=\left[\beta_{j 0}, \beta_{j 1}, \cdots, \beta_{j \hat{q}_{j}}\right] \in R^{r \times\left(\hat{q}_{j}+1\right)}, j=k+1, \cdots, r, \\
J_{j}=\left[\begin{array}{ccccc}
0 & 1 & 0 & \cdots & 0 \\
0 & 0 & 1 & \cdots & 0 \\
\cdot & \cdot & \cdot & \cdots & \cdot \\
0 & 0 & 0 & \cdots & 1 \\
0 & 0 & 0 & \cdots & 0
\end{array}\right] \in R^{\left.\left(\hat{q}_{j}+1\right) \times \hat{q}_{j}+1\right)}, \quad j=k+1, \cdots, r .
\end{gathered}
$$

We observe that

1. $J_{\infty}$ is nilpotent with index of nilpotency $\hat{q}_{r}+1$, because the largest Jordan block is $\left(\hat{q}_{r}+1\right) \times\left(\hat{q}_{r}+1\right)$.

2. $\mu=\sum_{j=k+1}^{r}\left(\hat{q}_{j}+1\right)=\sum_{j=k+1}^{r} \hat{q}_{j}+r-k$

The essential difference between these two interpretations of infinite Jordan pairs is the different roles which in particular the infinite poles and the infinite zeros play in system behaviours are concerned. It is obvious that $\nu \geq \mu$, and the dimensions of the generalised infinite Jordan pair of Definition 1 are unnecessaily larger than that of the infinite Jordan pair of Definition 2 due to some redundant information being included. This can be seen clearly from the following example.

Example 1 Consider the polynomial matrix

$$
A(s)=\left[\begin{array}{ccc}
1 & s^{3} & 0 \\
0 & 1 & s \\
0 & 0 & 1
\end{array}\right] \in R[s]^{3 \times 3}
$$

its Smith-McMillan form is

$$
S_{A(s)}^{\infty}=\left[\begin{array}{ccc}
s^{3} & 0 & 0 \\
0 & s & 0 \\
0 & 0 & \frac{1}{s^{4}}
\end{array}\right], \quad \text { i.e., } q_{1}=3, q_{2}=1, \hat{q}_{3}=4 .
$$

Thus $A(s)$ has 2 infinite poles at degrees 1 and 3 and an infinite zero at degree 4 . The dual polynomial matrix to $A(s)$ is

$$
D_{A(s)}(w)=w^{q_{1}} A\left(\frac{1}{w}\right)=\left[\begin{array}{ccc}
w^{3} & 1 & 0 \\
0 & w^{3} & w^{2} \\
0 & 0 & w^{3}
\end{array}\right] \in R[w]^{3 \times 3}
$$


and

$$
\tilde{U}_{L}(w) D_{A(s)}(w) \tilde{U}_{R}(w)=S_{D_{A(s)}(w)}^{0}=w^{q_{1}} S_{A(s)}^{\infty}\left(\frac{1}{w}\right)=\left[\begin{array}{ccc}
1 & 0 & 0 \\
0 & w^{2} & 0 \\
0 & 0 & w^{7}
\end{array}\right]
$$

is the local Smith form of $D_{A(s)}(w)$ at $w=0$, where

$$
\tilde{U}_{L}(w)=\left[\begin{array}{ccc}
1 & 0 & 0 \\
-w^{3} & 1 & 0 \\
w^{4} & -w & 1
\end{array}\right], \quad \tilde{U}_{R}(w)=\left[\begin{array}{ccc}
0 & 0 & 2 \\
1 & 0 & -w^{3} \\
0 & 1 & w^{4}
\end{array}\right]:=\left[u_{1}(w), u_{2}(w), u_{3}(w)\right] .
$$

So we get three kinds of infinite elementary divisor: $w^{2}$ corresponding to the infinite pole of $A(s), w^{7}$ corresponding to the infinite zero of $A(s)$ and the IED $1=w^{0}$. From

$$
\begin{aligned}
& \beta_{2 q}=\frac{1}{q !} u_{2}^{(q)}(0), \quad q=0,1 \\
& \beta_{3 q}=\frac{1}{q !} u_{3}^{(q)}(0), \quad q=0,1,2,3,4,5,6
\end{aligned}
$$

we have

$$
\begin{aligned}
& \beta_{20}=\left[\begin{array}{l}
0 \\
0 \\
1
\end{array}\right], \quad \beta_{21}=\left[\begin{array}{l}
0 \\
0 \\
0
\end{array}\right], \\
& \beta_{30}=\left[\begin{array}{l}
1 \\
0 \\
0
\end{array}\right], \quad \beta_{31}=\left[\begin{array}{l}
0 \\
0 \\
0
\end{array}\right], \quad \beta_{32}=\left[\begin{array}{l}
0 \\
0 \\
0
\end{array}\right], \\
& \beta_{33}=\left[\begin{array}{c}
0 \\
-1 \\
0
\end{array}\right], \quad \beta_{34}=\left[\begin{array}{l}
0 \\
0 \\
1
\end{array}\right], \quad \beta_{35}=\left[\begin{array}{l}
0 \\
0 \\
0
\end{array}\right], \quad \beta_{36}=\left[\begin{array}{l}
0 \\
0 \\
0
\end{array}\right] .
\end{aligned}
$$

According to Definition 1, the generalised infinite Jordan pair in the sense of Gohberg et al $(1982)$ is $\left(C_{\infty}^{\prime}, J_{\infty}^{\prime}\right)$ :

$$
\begin{aligned}
C_{\infty}^{\prime}= & {\left[C_{2}^{\prime}, C_{3}^{\prime}\right]=\left[\beta_{20}, \beta_{21}, \beta_{30}, \beta_{31}, \beta_{32}, \beta_{33}, \beta_{34}, \beta_{35}, \beta_{36}\right] } \\
= & {\left[\begin{array}{cc|ccccc|cc}
0 & 0 & 1 & 0 & 0 & 0 & 0 & 0 & 0 \\
0 & 0 & 0 & 0 & 0 & -1 & 0 & 0 & 0 \\
1 & 0 & 0 & 0 & 0 & 0 & 1 & 0 & 0
\end{array}\right] \in R^{r \times \nu} \quad(r=3, \nu=9), } \\
J_{\infty}^{\prime}= & {\left[\begin{array}{ll|lllll|ll}
0 & 1 & 0 & 0 & 0 & 0 & 0 & 0 & 0 \\
0 & 0 & 0 & 0 & 0 & 0 & 0 & 0 & 0 \\
\hline 0 & 0 & 0 & 1 & 0 & 0 & 0 & 0 & 0 \\
0 & 0 & 0 & 0 & 1 & 0 & 0 & 0 & 0 \\
0 & 0 & 0 & 0 & 0 & 1 & 0 & 0 & 0 \\
0 & 0 & 0 & 0 & 0 & 0 & 1 & 0 & 0 \\
0 & 0 & 0 & 0 & 0 & 0 & 0 & 1 & 0 \\
\hline 0 & 0 & 0 & 0 & 0 & 0 & 0 & 0 & 1 \\
0 & 0 & 0 & 0 & 0 & 0 & 0 & 0 & 0
\end{array}\right] \in R^{9 \times 9}, }
\end{aligned}
$$


While the infinite Jordan pair in the sense of Vardulakis $(1991)$ is $\left(C_{\infty}, J_{\infty}\right)$ with:

$$
\begin{aligned}
C_{\infty}= & {\left[C_{3}\right]=\left[\beta_{30}, \beta_{31}, \beta_{32}, \beta_{33}, \beta_{34}\right] } \\
= & {\left[\begin{array}{ccccc}
1 & 0 & 0 & 0 & 0 \\
0 & 0 & 0 & -1 & 0 \\
0 & 0 & 0 & 0 & 1
\end{array}\right] \in R^{r \times \mu} \quad\left(r=3, \mu=\hat{q}_{3}+1=5\right), } \\
J_{\infty}= & {\left[\begin{array}{lllll}
0 & 1 & 0 & 0 & 0 \\
0 & 0 & 1 & 0 & 0 \\
0 & 0 & 0 & 1 & 0 \\
0 & 0 & 0 & 0 & 1 \\
0 & 0 & 0 & 0 & 0
\end{array}\right] \in R^{5 \times 5} . }
\end{aligned}
$$

In the above example it is noted that the dimensions of the generalised infinite Jordan pair which are $3 \times 9$ and $9 \times 9$ are unnecessarily larger than that of the infinite Jordan pair which are $3 \times 5$ and $5 \times 5$. Such tendency will become more evident as the number of the infinite poles of $A(s)$, the dimension of $A(s)$ and the degree of $A(s)$ are increased. However, the following observation arising from the above two definitions of infinite Jordan pairs suggests the possibility of deleting the redundant information which is contained in the generalised infinite Jordan pair.

Theorem 1 If $\left(C_{\infty}^{\prime}, J_{\infty}^{\prime}\right)$ is a generalized infinite Jordan pair, there exists an elementary matrix $P$ such that

$$
\begin{aligned}
C_{\infty}^{\prime} P & =\left[C_{\infty}, C_{0}\right] \\
P^{-1} J_{\infty}^{\prime} P & =\left[\begin{array}{c|c}
J_{\infty} & J^{\prime} \\
\hline 0 & J_{0}
\end{array}\right]
\end{aligned}
$$

where $C_{0} \in R^{r \times(\nu-\mu)}, J^{\prime} \in R^{\mu \times(\nu-\mu)}, J_{0} \in R^{(\nu-\mu) \times(\nu-\mu)}, 0 \in R^{(\nu-\mu) \times \mu}$, and $\left(C_{\infty}, J_{\infty}\right)$ is an infinite Jordan pair. Furthermore, $J_{0}$ is nilpotent with index of nilpotency $q_{1}-1$.

Proof: Note that $C_{\infty}$ is a submatrix of $C_{\infty}^{\prime}$. By a series of interchanging of two columns in $C_{\infty}^{\prime}, C_{\infty}^{\prime}$ can be brought to the form $\left[C_{\infty}, C_{0}\right]$. We denote this elementary matrix as $P$, then $P^{-1}=P^{T}, C_{\infty}^{\prime} P=\left[C_{\infty}, C_{0}\right]$, where $C_{0} \in R^{r \times(\nu-\mu)}$. Similarly, by interchanging the corresponding columns and the corresponding rows of $J_{\infty}^{\prime}, J_{\infty}^{\prime}$ can be brought to a block matrix, i.e.,

$$
P^{-1} J_{\infty}^{\prime} P=\left[\begin{array}{c|c}
J_{\infty} & J^{\prime} \\
\hline 0 & J_{0}
\end{array}\right]
$$

where

$$
\begin{aligned}
& J^{\prime}= {\left[a\left(\sigma_{1}, \sigma_{2}\right)\right]_{\left(\sigma_{1}, \sigma_{2}\right)} \in R^{\mu \times(\nu-\mu)}, } \\
& a\left(\sigma_{1}, \sigma_{2}\right)=\left\{\begin{array}{cc}
1 & \sigma_{1}=\sum_{\sigma=k+1}^{j}\left(\hat{q}_{\sigma}+1\right), \sigma_{2}=\sum_{j=2}^{k} \tau(j)+\left(\hat{q}_{j}+2\right), j=k+1, \cdots, r \\
0 & \text { other }
\end{array}\right. \\
& J_{0}= \text { block diag }\left[J_{2}^{\prime}, \cdots, J_{k}^{\prime}, J_{k+1}^{\prime}, \cdots, J_{r}^{\prime}\right], \\
& J_{j}^{\prime}(j=2,3, \cdots, k) \text { are Jordan matrix of }\left(q_{1}-q_{j}\right) \times\left(q_{1}-q_{j}\right)(j=2, \cdots, k), \\
& J_{i}^{\prime} \quad(i=k+1, \cdots, r) \text { are Jordan matrix of }\left(q_{1}-1\right) \times\left(q_{1}-1\right) .
\end{aligned}
$$

It can easily be seen that $J_{0}$ is nilpotent with index of nilpotency $q_{1}-1$. 
Remark 1 The above elementary matrix $P$ has the effect of deleting the redundant information in two ways. First, it deletes the redundant information in those blocks in the infinite Jordan pair of Gohberg et al (1982) which corresponds to the infinite zeros and bring them into the correct sizes. Second, it deletes the whole blocks in $C_{\infty}^{\prime}, J_{\infty}^{\prime}$ which corresponds to the infinite poles and the whole blocks which are not dynamically important. In this way the resulting resolvent decomposition more precisely reflects the relevant system structure. This will be seen explicitly when the effect of $P$ on $Z$ has been determined in a subsequent result.

Example 2 Recall Example 1, we have

$$
\begin{aligned}
& \underbrace{\left[\begin{array}{rrrrrrrrr}
0 & 0 & 1 & 0 & 0 & 0 & 0 & 0 & 0 \\
0 & 0 & 0 & 0 & 0 & -1 & 0 & 0 & 0 \\
1 & 0 & 0 & 0 & 0 & 0 & 1 & 0 & 0
\end{array}\right]}_{C_{\infty}^{\prime}} \underbrace{\left[\begin{array}{rrrrrrrrr}
0 & 0 & 0 & 0 & 0 & 1 & 0 & 0 & 0 \\
0 & 0 & 0 & 0 & 0 & 0 & 1 & 0 & 0 \\
1 & 0 & 0 & 0 & 0 & 0 & 0 & 0 & 0 \\
0 & 1 & 0 & 0 & 0 & 0 & 0 & 0 & 0 \\
0 & 0 & 1 & 0 & 0 & 0 & 0 & 0 & 0 \\
0 & 0 & 0 & 1 & 0 & 0 & 0 & 0 & 0 \\
0 & 0 & 0 & 0 & 1 & 0 & 0 & 0 & 0 \\
0 & 0 & 0 & 0 & 0 & 0 & 0 & 1 & 0 \\
0 & 0 & 0 & 0 & 0 & 0 & 0 & 0 & 1
\end{array}\right]}_{P}
\end{aligned}
$$

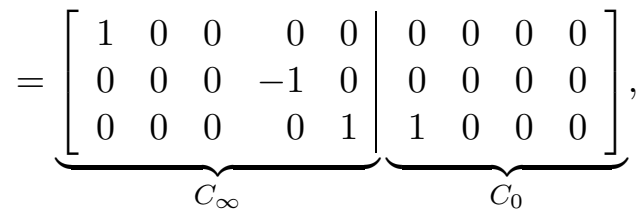$$
\underbrace{\left[\begin{array}{lllllllll}
0 & 0 & 1 & 0 & 0 & 0 & 0 & 0 & 0 \\
0 & 0 & 0 & 1 & 0 & 0 & 0 & 0 & 0 \\
0 & 0 & 0 & 0 & 1 & 0 & 0 & 0 & 0 \\
0 & 0 & 0 & 0 & 0 & 1 & 0 & 0 & 0 \\
0 & 0 & 0 & 0 & 0 & 0 & 1 & 0 & 0 \\
1 & 0 & 0 & 0 & 0 & 0 & 0 & 0 & 0 \\
0 & 1 & 0 & 0 & 0 & 0 & 0 & 0 & 0 \\
0 & 0 & 0 & 0 & 0 & 0 & 0 & 1 & 0 \\
0 & 0 & 0 & 0 & 0 & 0 & 0 & 0 & 1
\end{array}\right]}_{P^{-1}} \underbrace{\left[\begin{array}{lllllllll}
0 & 1 & 0 & 0 & 0 & 0 & 0 & 0 & 0 \\
0 & 0 & 0 & 0 & 0 & 0 & 0 & 0 & 0 \\
0 & 0 & 0 & 1 & 0 & 0 & 0 & 0 & 0 \\
0 & 0 & 0 & 0 & 1 & 0 & 0 & 0 & 0 \\
0 & 0 & 0 & 0 & 0 & 1 & 0 & 0 & 0 \\
0 & 0 & 0 & 0 & 0 & 0 & 1 & 0 & 0 \\
0 & 0 & 0 & 0 & 0 & 0 & 0 & 1 & 0 \\
0 & 0 & 0 & 0 & 0 & 0 & 0 & 0 & 1 \\
0 & 0 & 0 & 0 & 0 & 0 & 0 & 0 & 0
\end{array}\right]}_{J_{\infty}^{\prime}} \times
$$$$
\times \underbrace{\left[\begin{array}{ccccccccc}
0 & 0 & 0 & 0 & 0 & 1 & 0 & 0 & 0 \\
0 & 0 & 0 & 0 & 0 & 0 & 1 & 0 & 0 \\
1 & 0 & 0 & 0 & 0 & 0 & 0 & 0 & 0 \\
0 & 1 & 0 & 0 & 0 & 0 & 0 & 0 & 0 \\
0 & 0 & 1 & 0 & 0 & 0 & 0 & 0 & 0 \\
0 & 0 & 0 & 1 & 0 & 0 & 0 & 0 & 0 \\
0 & 0 & 0 & 0 & 1 & 0 & 0 & 0 & 0 \\
0 & 0 & 0 & 0 & 0 & 0 & 0 & 1 & 0 \\
0 & 0 & 0 & 0 & 0 & 0 & 0 & 0 & 1
\end{array}\right]}_{P}
$$ 


$$
=\left[\begin{array}{lllll|llll}
0 & 1 & 0 & 0 & 0 & 0 & 0 & 0 & 0 \\
0 & 0 & 1 & 0 & 0 & 0 & 0 & 0 & 0 \\
0 & 0 & 0 & 1 & 0 & 0 & 0 & 0 & 0 \\
0 & 0 & 0 & 0 & 1 & 0 & 0 & 0 & 0 \\
0 & 0 & 0 & 0 & 0 & 0 & 0 & 1 & 0 \\
\hline 0 & 0 & 0 & 0 & 0 & 0 & 1 & 0 & 0 \\
0 & 0 & 0 & 0 & 0 & 0 & 0 & 0 & 0 \\
0 & 0 & 0 & 0 & 0 & 0 & 0 & 0 & 1 \\
0 & 0 & 0 & 0 & 0 & 0 & 0 & 0 & 0
\end{array}\right]:=\left[\begin{array}{c|c}
J_{\infty} & J^{\prime} \\
\hline 0 & J_{0}
\end{array}\right] .
$$

It is noted that here $J_{0}$ is nilpotent with index of nilpotency 2 .

The key point in here is to find the above elementary matrix $P$ to delete the redundant information. Based on the information carried by the Smith-McMillan form at $s=\infty$ of $A(s), P$ results from a series of elementary operations from an identity matrix, which is very easy to obtain. Once the redundant information is deleted from the generalised infinite Jordan pair, the generalised infinite Jordan pair and the elementary matrix $P$ are not involved in computation in the solution procedure.

\section{The Solution of Regular PMDs}

We consider now the homogeneous regular PMD:

$$
A(\rho) \xi(t)=0, \quad t \geq 0 .
$$

Let $S_{A(s)}^{\infty}(s)$ be the Smith-McMillan form at $s=\infty$ of $A(s)=L_{-}[A(\rho)]=A_{0}+A_{1} s+$ $\cdots+A_{q_{1}} s^{q_{1}}$ :

$$
S_{A(s)}^{\infty}(s)=\operatorname{diag}\left[s^{q_{1}}, s^{q_{2}}, \cdots, s^{q_{k}}, \frac{1}{s^{\hat{q}_{k+1}}}, \cdots, \frac{1}{s^{\hat{q}_{r}}}\right] \in R^{r \times r}(s)
$$

and if $A(s)$ has at least one zero at $s=\infty$ then the Laurent expansion of $A^{-1}(s)$ can be written as follows

$$
\begin{aligned}
A^{-1}(s) & =H_{\hat{q}_{r}} s^{\hat{q}_{r}}+H_{\hat{q}_{r}-1} s^{\hat{q}_{r}-1}+\cdots+H_{1} s+H_{0}+H_{-1} s^{-1}+H_{-2} s^{-2}+\cdots \\
& =H_{p o l}(s)+H_{s p}(s)
\end{aligned}
$$

where $H_{p o l}(s) \in R^{r \times r}[s]$ is the polynomial part of $A^{-1}(s)$ and $H_{s p}(s) \in R^{r \times r}(s)$ is the strictly proper part of $A^{-1}(s)$. Let a resolvent decomposition of $A(s)$ be given by

$$
A^{-1}(s)=C_{\infty}\left(s J_{\infty}-I_{\mu}\right)^{-1} Z_{2}+C\left(s I_{n}-J\right)^{-1} Z_{1}
$$

where $n:=\operatorname{deg} \operatorname{det}(A(s))$. It should be noted that $\left(C, J, Z_{1}\right)$ is a realization of $H_{s p}(s)$, and $\left(C_{\infty}, J_{\infty}, Z_{2}\right)$ is a realization of $H_{p o l}(s)$. Considering the $L_{-}$Laplace transformation of equation (4), we obtain

$$
\hat{\xi}(s)=A^{-1}(s) \hat{\alpha}(s) \in R^{r \times 1}
$$


where $\hat{\alpha}(s) \in R^{r \times 1}[s]$ is the initial condition vector associated with the initial values of $\xi(t)$ and its $\left(q_{1}-1\right)$-derivatives at $t=0-$, i.e., $\xi(0-), \xi^{(1)}(0-), \cdots, \xi^{\left(q_{1}-1\right)}(0-)$ given by Fossard (1972), Pugh (1976)

$$
\hat{\alpha}(s)=\left[s^{q_{1}-1} I_{r}, s^{q_{1}-2} I_{r}, \cdots, s I_{r}, I_{r}\right]\left[\begin{array}{cccc}
A_{q_{1}} & 0 & \cdots & 0 \\
A_{q_{1}-1} & A_{q_{1}} & \cdots & 0 \\
\vdots & \vdots & \ddots & \vdots \\
A_{1} & A_{2} & \cdots & A_{q_{1}}
\end{array}\right]\left[\begin{array}{c}
\xi(0-) \\
\xi^{(1)}(0-) \\
\vdots \\
\xi^{\left(q_{1}-1\right)}(0-)
\end{array}\right] .
$$

We obtain

$$
\hat{\xi}(s)=\left[C, C_{\infty}\right]\left[\begin{array}{c|c}
s I_{n}-J & 0_{n, \mu} \\
\hline 0_{\mu, n} & s J_{\infty}-I_{\mu}
\end{array}\right]^{-1}\left[\begin{array}{c}
x_{s}(0-) \\
J_{\infty} x_{f}(0-)
\end{array}\right]
$$

where the vector

$$
\begin{aligned}
& {\left[\begin{array}{c}
x_{s}(0-) \\
J_{\infty} x_{f}(0-)
\end{array}\right]=\left[\begin{array}{c|c}
J^{q_{1}-1} Z_{1}, \cdots, Z_{1} & 0_{n, q_{1} \mu} \\
\hline 0_{\mu, q_{1} n} & J_{\infty} Z_{2}, \cdots, J_{\infty}^{q_{1}} Z_{2}
\end{array}\right]} \\
& {\left[\begin{array}{cccc}
A_{q_{1}} & 0 & \cdots & 0 \\
A_{q_{1}-1} & A_{q_{1}} & \cdots & 0 \\
\vdots & \vdots & \ddots & \vdots \\
A_{1} & A_{2} & \cdots & A_{q_{1}} \\
\hline A_{0} & A_{1} & \cdots & A_{q_{1}-1} \\
0 & A_{0} & \cdots & A_{q_{1}-2} \\
\vdots & \vdots & \ddots & \vdots \\
0 & 0 & \cdots & A_{0}
\end{array}\right]\left[\begin{array}{c}
\xi(0-) \\
\xi^{(1)}(0-) \\
\vdots \\
\xi^{\left(q_{1}-1\right)}(0-)
\end{array}\right] \in R^{(n+\mu) \times 1} .}
\end{aligned}
$$

Now by taking the inverse $L_{-}$Laplace transformation of (7), we have the following result.

Lemma 1 (Vardulakis, 1991) The general solution of homogeneous PMD (4) is

$$
\xi(t)=C e^{J t} x_{s}(0-)-C_{\infty} \sum_{i=1}^{\hat{q}_{r}} \delta(t)^{(i-1)} J_{\infty}^{i-1}\left(J_{\infty} x_{f}(0-)\right)
$$

where $\delta(t)$ is the Dirac impulse function.

The next result constructs a resolvent decomposition for a regular polynomial matrix, which follows from Gohberg et al (1982).

Lemma 2 Let $A(s)=A_{0}+A_{1} s+\cdots+A_{q_{1}} s^{q_{1}} \in R^{r \times r}[s], \operatorname{rank}_{\Re[s]} A(s)=r$. If $(C, J)$ is the finite Jordan pair of $A(s)$, and $\left(C_{\infty}^{\prime}, J_{\infty}^{\prime}\right)$ is the generalised infinite Jordan pair of $A(s)$ in the sense of Gohberg et al (1982), $n:=\operatorname{deg} \operatorname{det}(A(s))$. Put

$$
\begin{aligned}
V & =\left[A_{q_{1}} C J^{q_{1}-1},-\sum_{i=0}^{q_{1}-1} A_{i} C_{\infty}^{\prime}\left(J_{\infty}^{\prime}\right)^{q_{1}-1-i}\right], \\
S & =\left[\begin{array}{cc}
C & C_{\infty}^{\prime}\left(J_{\infty}^{\prime}\right)^{q_{1}-2} \\
C J & C_{\infty}^{\prime}\left(J_{\infty}^{\prime}\right)^{q_{1}-3} \\
\vdots & \vdots \\
C J^{q_{1}-2} & C_{\infty}^{\prime}
\end{array}\right],
\end{aligned}
$$


and

$$
Z^{\prime}:=\left[\begin{array}{c}
Z_{1} \\
Z_{2}^{\prime}
\end{array}\right]=\left[\begin{array}{c|c}
I_{n} & 0 \\
\hline 0 & \left(J_{\infty}^{\prime}\right)^{q_{1}-1}
\end{array}\right]\left[\begin{array}{c}
S \\
V
\end{array}\right]^{-1}[0, \cdots, 0, I]^{T}
$$

then

$$
A^{-1}(s)=\left[C, C_{\infty}^{\prime}\right]\left[\begin{array}{c|c}
I_{n} s-J & 0 \\
\hline 0 & J_{\infty}^{\prime} s-I_{\nu}
\end{array}\right]^{-1}\left[\begin{array}{c}
Z_{1} \\
Z_{2}^{\prime}
\end{array}\right]
$$

An interesting observation from (9) is the following.

Proposition 1 If we partition

$$
Z^{\prime}:=\left[\begin{array}{c}
Z_{1} \\
Z_{2}^{\prime}
\end{array}\right]:=\left[\begin{array}{c}
Z_{1} \\
Z_{21}^{\prime} \\
Z_{22}^{\prime}
\end{array}\right]
$$

where $Z_{1} \in R^{n \times r}, Z_{21}^{\prime} \in R^{\mu \times r}, Z_{22}^{\prime} \in R^{(\nu-\mu) \times r}$, then

$$
\left[\begin{array}{c|c}
I_{n} & 0 \\
\hline 0 & P^{-1}
\end{array}\right]\left[\begin{array}{c}
Z_{1} \\
\hline Z_{21}^{\prime} \\
Z_{22}^{\prime}
\end{array}\right]=\left[\begin{array}{c}
Z_{1} \\
Z_{2} \\
0
\end{array}\right]
$$

where $Z_{2} \in R^{\mu \times r}$.

Proof : Let

$$
\left[\begin{array}{c}
\Pi_{1} \\
\Pi_{2} \\
\Pi_{3}
\end{array}\right]:=\left[\begin{array}{c}
S \\
V
\end{array}\right]^{-1}[0, \cdots, 0, I]^{T}
$$

where $\Pi_{1} \in R^{n \times 1}, \Pi_{2} \in R^{\mu \times 1}, \Pi_{3} \in R^{(\nu-\mu) \times 1}$. From Theorem 1, there exists an elementary matrix $P \in R^{\nu \times \nu}$, such that

$$
J_{\infty}^{\prime}=P\left[\begin{array}{c|c}
J_{\infty} & J^{\prime} \\
\hline 0 & J_{0}
\end{array}\right] P^{-1}
$$

So

$$
\left(J_{\infty}^{\prime}\right)^{q_{1}-1}=P\left[\begin{array}{c|c}
\left(J_{\infty}\right)^{q_{1}-1} & Q \\
\hline 0 & \left(J_{0}\right)^{q_{1}-1}
\end{array}\right] P^{-1},
$$

where $Q=J_{\infty}^{q_{1}-2} J^{\prime}+J_{\infty}^{q_{1}-3} J^{\prime} J_{0}+\cdots+J^{\prime} J_{0}^{q_{1}-2}$.

$$
Z^{\prime}=\left[\begin{array}{c|c}
I_{n} & 0 \\
\hline 0 & P
\end{array}\right]\left[\begin{array}{c|cc}
I_{n} & 0 & 0 \\
\hline 0 & J_{\infty}^{q_{1}-1} & Q \\
0 & 0 & J_{0}^{q_{1}-1}
\end{array}\right]\left[\begin{array}{c|c}
I_{n} & 0 \\
\hline 0 & P^{-1}
\end{array}\right]\left[\begin{array}{c}
\Pi_{1} \\
\Pi_{2} \\
\Pi_{3}
\end{array}\right]:=\left[\begin{array}{c}
Z_{1} \\
Z_{21}^{\prime} \\
Z_{22}^{\prime}
\end{array}\right] .
$$

Noticing $J_{0}$ is nilpotent with index of nilpotency $q_{1}-1$, i.e., $J_{0}^{q_{1}-1}=0$, it follows that

$$
\left[\begin{array}{c|c}
I_{n} & 0 \\
\hline 0 & P^{-1}
\end{array}\right]\left[\begin{array}{c}
Z_{1} \\
\hline Z_{21}^{\prime} \\
Z_{22}^{\prime}
\end{array}\right]=\left[\begin{array}{c}
Z_{1} \\
Z_{2} \\
0
\end{array}\right] .
$$


The above observation is interesting, not least for the way in which the redundant information contained in $Z^{\prime}$ is deleted. More importantly than this, however, is that such mechanism of decoupling makes the computation of $P$ attractive and facilitates the proposed refined resolvent decomposition.

The following result proposes a new approach to construct a resolvent decomposition in terms of the finite Jordan pair, the infinite Jordan pair and the generalised infinite Jordan pair, which is fundamental for us to formulate the solution of the PMD. As one of our main results, we are ready to state it as follows.

Theorem 2 If $(C, J)$ is the finite Jordan pair of $A(s)$ and $\left(C_{\infty}, J_{\infty}\right)$ is the infinite Jordan pair of $A(s)$ in the sense of Vardulakis (1991), $Z^{\prime}:=\left[\begin{array}{c}Z_{1} \\ Z_{2}^{\prime}\end{array}\right]:=\left[\begin{array}{c}Z_{1} \\ Z_{21}^{\prime} \\ Z_{22}^{\prime}\end{array}\right]$, is given by Lemma 2 , let $Z_{2} \in R^{\mu \times r}$ be given by

$$
\left[\begin{array}{c}
Z_{2} \\
0
\end{array}\right]=P^{-1}\left[\begin{array}{l}
Z_{21}^{\prime} \\
Z_{22}^{\prime}
\end{array}\right]
$$

then

$$
A^{-1}(s)=\left[C, C_{\infty}\right]\left[\begin{array}{c|c}
I_{n} s-J & 0 \\
\hline 0 & J_{\infty} s-I_{\mu}
\end{array}\right]^{-1}\left[\begin{array}{c}
Z_{1} \\
Z_{2}
\end{array}\right] .
$$

Proof: This follows readily from (10) and Theorem 1 on noting that

$$
\begin{aligned}
C_{\infty}^{\prime}\left(J_{\infty}^{\prime} s-I_{\nu}\right)^{-1} Z_{2}^{\prime} & =\left[C_{\infty}, C_{0}\right] P^{-1} P\left[\begin{array}{c|c}
s J_{\infty}-I_{\mu} & s J^{\prime} \\
\hline 0 & s J_{0}-I_{\nu-\mu}
\end{array}\right]^{-1} P^{-1} P\left[\begin{array}{c}
Z_{2} \\
0
\end{array}\right] \\
& =C_{\infty}\left(J_{\infty} s-I_{\mu}\right)^{-1} Z_{2}
\end{aligned}
$$

Remark 2 The resolvent decomposition proposed by Vardulakis (1991) reflects the system solution structure precisely, however, the realization approach is not so straightforward by itself. On the other hand in the resolvent decomposition proposed by Gohberg et al. (1982) (Lemma 2), $Z_{1}, Z_{2}^{\prime}$ were formulated explicitly. However, there is some redundant information as described in Theorem 1 and it does not give such a precise insight into the system structure. The resolvent decomposition proposed here is a refinement of these two results and inherits the advantages of both results. Due to the fact that the redundant information is deleted, the proposed refined resolvent decomposition has minimal dimensions, the proposed approach has an obvious advantage, i.e., computation of the inverse matrix of $A(s)$ can be carried out more efficiently.

For the nonhomogeneous regular PMD

$$
A(\rho) \xi(t)=B u(t), \quad t \geq 0
$$

the following theorem gives the complete solution. 
Theorem 3 Let $A(s)=A_{0}+A_{1} s+\cdots+A_{q_{1}} s^{q_{1}} \in R^{r \times r}[s]$ be regular, and let $S_{A(s)}^{\infty}(s)=$ $\operatorname{diag}\left[s^{q_{1}}, s^{q_{2}}, \cdots, s^{q_{k}}, 1 / s^{\hat{q}_{k+1}}, \cdots, 1 / s^{\hat{q}_{r}}\right]$ be the Smith-McMillan form of $A(s)$ at $s=\infty$. If $(C, J)$ is the finite Jordan pair of $A(s)$ and $\left(C_{\infty}, J_{\infty}\right)$ is the infinite Jordan pair of $A(s)$ (in the sense of Vardulakis, 1991), $\left(C, J, Z_{1}\right)$ and $\left(C_{\infty}, J_{\infty}, Z_{2}\right)$ satisfy the resolvent decomposition (11), then the complete solution of regular PMD (12) is

$$
\begin{aligned}
\xi(t)=\quad & C e^{J t} x_{s}(0-)+C \int_{0-}^{t} e^{J(t-\tau)} Z_{1} B u(\tau) d \tau \\
& -C_{\infty} \sum_{i=1}^{\hat{q}_{r}} \delta(t)^{(i-1)} J_{\infty}^{i-1}\left(J_{\infty} x_{f}(0-)\right)-C_{\infty} \sum_{i=0}^{\hat{q}_{r}} J_{\infty}^{i} Z_{2} B u^{(i)}(t)
\end{aligned}
$$

where $x_{s}(0-), J_{\infty} x_{f}(0-)$ are initial values given by $(8)$.

Proof: From Lemma 1, one knows that

$$
\xi(t)=C e^{J t} x_{s}(0-)-C_{\infty} \sum_{i=1}^{\hat{q}_{r}} \delta(t)^{(i-1)} J_{\infty}^{i-1}\left(J_{\infty} x_{f}(0-)\right)
$$

generates the general solution of the homogeneous PMD, so we only need to check that the formula (13) does indeed produce a solution of the nonhomogeneous PMD (12). To this end, when $t>0$, we have

$$
\begin{aligned}
\xi^{(j)}(t)=\quad & C J^{j} e^{J t} x_{s}(0-)+\sum_{k=0}^{j-1} C J^{j-1-k} Z_{1} B u^{(k)}(t) \\
& +C \int_{0-}^{t} J^{j} e^{J(t-\tau)} Z_{1} B u(\tau) d \tau-C_{\infty} \sum_{i=0}^{\hat{q}_{r}} J_{\infty}^{i} Z_{2} B u^{(i+j)}(t),
\end{aligned}
$$

SO

$$
\begin{aligned}
A(\rho) \xi(t)= & \sum_{j=0}^{q_{1}} A_{j} \xi^{(j)}(t) \\
= & \sum_{j=0}^{q_{1}} A_{j} C J^{j} e^{J t} x_{s}(0-)+\sum_{j=0}^{q_{1}} \sum_{k=0}^{j-1} A_{j} C J^{j-1-k} Z_{1} B u^{(k)}(t) \\
& +\int_{0-}^{t}\left[\sum_{j=0}^{q_{1}} A_{j} C J^{j}\right] e^{J(t-\tau)} Z_{1} B u(\tau) d \tau-\sum_{i=0}^{\hat{q}_{r}} \sum_{j=0}^{q_{1}} A_{j} C_{\infty} J_{\infty}^{i} Z_{2} B u^{(i+j)}(t) .
\end{aligned}
$$

Using the property $\sum_{j=0}^{q_{1}} A_{j} C J^{j}=0$ and rearranging the terms, we have

$$
\begin{aligned}
A(\rho) \xi(t) & =\sum_{j=0}^{q_{1}} A_{j} \xi^{(j)}(t) \\
& =\sum_{k=0}^{q_{1}+\hat{q}_{r}}\left\{\sum_{j=k+1}^{q_{1}} A_{j} C J^{j-1-k} Z_{1}-\sum_{j=0}^{k} A_{j} C_{\infty} J_{\infty}^{k-j} Z_{2}\right\} B u^{(k)}(t) .
\end{aligned}
$$

Noticing

$$
\begin{aligned}
A^{-1}(s)= & {\left[C, C_{\infty}\right]\left[\begin{array}{c|c}
\left(I_{n} s-J\right)^{-1} & 0 \\
0 & \left(J_{\infty} s-I_{\mu}\right)^{-1}
\end{array}\right]\left[\begin{array}{c}
Z_{1} \\
Z_{2}
\end{array}\right] } \\
= & C_{\infty}\left(J_{\infty} s-I\right)^{-1} Z_{2}+C(I s-J)^{-1} Z_{1} \\
= & -C_{\infty} J_{\infty}^{\hat{q}_{r}} Z_{2} s^{\hat{q}_{r}}-\cdots-C_{\infty} J_{\infty} Z_{2} s-C_{\infty} Z_{2} \\
& +C Z_{1} s^{-1}+C J Z_{1} s^{-2}+\cdots,
\end{aligned}
$$


from

$$
A(s) A^{-1}(s)=I
$$

it follows that

$$
\sum_{j=k+1}^{q_{1}} A_{j} C J^{j-1-k} Z_{1}-\sum_{j=0}^{k} A_{j} C_{\infty} J_{\infty}^{k-j} Z_{2}=\left\{\begin{array}{ll}
I, & k=0 \\
0, & k>0
\end{array} .\right.
$$

Hence

$$
A(\rho) \xi(t)=B u(t)
$$

which finishes the proof.

Remark 3 The above solution displays precisely the zero state response by means of

$$
C \int_{0-}^{t} e^{J(t-\tau)} Z_{1} B u(\tau) d \tau-C_{\infty} \sum_{i=0}^{\hat{q}_{r}} J_{\infty}^{i} Z_{2} B u^{(i)}(t)
$$

and the zero input response through the term

$$
C e^{J t} x_{s}(0-)-C_{\infty} \sum_{i=1}^{\hat{q}_{r}} \delta(t)^{(i-1)} J_{\infty}^{i-1}\left(J_{\infty} x_{f}(0-)\right) .
$$

Also the impulsive properties of the system are displayed in this solution due to the fact that the initial conditions of the pseudo state are considered. Such impulsive solution part to the general nonhomogeneous PMDs is however unavailable from the solution of Gohberg et al (1982) and Vardulakis (1991).

Remark 4 According to Gohberg et al (1982), the solution of PMD (12) is following (without the impulse solution part):

$$
\xi(t)=C e^{J t} x_{s}(0-)+C \int_{0-}^{t} e^{J(t-\tau)} Z_{1} B u(\tau) d \tau-C_{\infty}^{\prime} \sum_{i=0}^{\hat{q}_{r}+q_{1}-1}\left(J_{\infty}^{\prime}\right)^{i} Z_{2}^{\prime} B u^{(i)}(t)
$$

where $\left(C_{\infty}^{\prime}, J_{\infty}^{\prime}\right)$ is the infinite Jordan pairs in the sense of Gohberg et al (1982). $Z_{1}, Z_{2}^{\prime}$ are given by (9). By using Theorem 1, Proposition 1 and noticing that $J_{\infty}$ is nilpotent with index of nilpotency $\hat{q}_{r}+1$, one finds that

$$
\begin{aligned}
\sum_{i=0}^{\hat{q}_{r}+q_{1}-1} C_{\infty}^{\prime}\left(J_{\infty}^{\prime}\right)^{i} Z_{2}^{\prime} B u^{(i)}(t) & =\sum_{i=0}^{\hat{q}_{r}+q_{1}-1}\left[C_{\infty}, C_{0}\right] P^{-1} P\left[\begin{array}{c|c}
J_{\infty} & J^{\prime} \\
\hline 0 & J_{0}
\end{array}\right]^{i} P^{-1} P\left[\begin{array}{c}
Z_{2} \\
0
\end{array}\right] B u^{(i)}(t) \\
& =\sum_{i=0}^{\hat{q}_{r}} C_{\infty} J_{\infty}^{i} Z_{2} B u^{(i)}(t)
\end{aligned}
$$

It is thus clearly seen that the redundant information included in the solution of Gohberg et al (1982) due to using the generalised infinite Jordan pairs does not appear in our solution any more. Also this helps to clarify the mechanism of decoupling in the solution of Gohberg et al (1982).

By comparing our solution with the solution of Gohberg et al (1982) and the solution of Vardulakis (1991), it can be seen that, besides the fact that our solution displays the impulsive solution part, our solution can be carried out more efficiently in actual computation, for the refined resolvent decomposition does not contain any redundant information, the infinite Jordan pairs used in our solution are of minimal dimensions, the matrices $Z_{1}$ and $Z_{2}$ are formulated explicitly. 


\section{Algorithm and example}

As mentioned before, the refined resolvent decomposition plays a key role in our approach, the difficulty in obtaining the solution of the regular PMDs depends on the calculation of this refined resolvent decomposition. To help clarify the construction of this refined resolvent decomposition, for the implementation of Theorem 2, the following algorithm is now given, which is useful for symbolic computational packages Maple.

Algorithm 1 (Computation of the refined resolvent decomposition of $A(s) \in R[s]^{r \times r}$ )

Step 1: Let $A(s)=\sum_{i=0}^{q_{1}} A_{i} s^{i}$, calculate the Smith form and the finite Jordan pair $(C, J)$.

Step 2: Find the dual polynomial $D_{A(s)}(w)$ of $A(s)$, calculate the local Smith form of $D_{A(s)}(w)$ at $w=0 S_{D_{A(s)}}^{0}(w)$ and the unimodular matrices $\tilde{U}_{L}(w), \tilde{U}_{R}(w)$ such that

$$
\tilde{U}_{L}(w) D_{A(s)}(w) \tilde{U}_{R}(w)=S_{D_{A(s)}}^{0}(w) .
$$

Step 3: Differentiate the matrix $\tilde{U}_{R}(w)$ and then construct the generalised infinite Jordan pair $\left(C_{\infty}^{\prime}, J_{\infty}^{\prime}\right)$ and the infinite Jordan pair $\left(C_{\infty}, J_{\infty}\right)$.

Step 4: Find the elementary matrix $P$ by using Theorem 1.

Step 5: Calculate $V, S$ and $Z^{\prime}$ by Lemma 2 .

Step 6: Partition $Z^{\prime}=\left[\begin{array}{c}Z_{1} \\ Z_{2}^{\prime}\end{array}\right]=\left[\begin{array}{c}Z_{1} \\ Z_{21}^{\prime} \\ Z_{22}^{\prime}\end{array}\right]$, from $\left[\begin{array}{c}Z_{2} \\ 0\end{array}\right]=P^{-1}\left[\begin{array}{c}Z_{21}^{\prime} \\ Z_{22}^{\prime}\end{array}\right]$ calculate $Z_{2}$.

Step 7: Finally obtain the refined resolvent decomposition

$$
A^{-1}(s)=C\left(s I_{n}-J\right)^{-1} Z_{1}+C_{\infty}\left(s J_{\infty}-I_{\mu}\right)^{-1} Z_{2} .
$$

Example 3 Let

$$
A(s)=\left[\begin{array}{ccc}
1 & s^{3} & 0 \\
0 & 1 & s \\
0 & 0 & s^{2}
\end{array}\right]:=A_{0}+A_{1} s+A_{2} s^{2}+A_{3} s^{3}
$$

with

$$
\begin{array}{ll}
A_{0}=\left[\begin{array}{lll}
1 & 0 & 0 \\
0 & 1 & 0 \\
0 & 0 & 0
\end{array}\right], & A_{1}=\left[\begin{array}{lll}
0 & 0 & 0 \\
0 & 0 & 1 \\
0 & 0 & 0
\end{array}\right], \\
A_{2}=\left[\begin{array}{lll}
0 & 0 & 0 \\
0 & 0 & 0 \\
0 & 0 & 1
\end{array}\right], & A_{3}=\left[\begin{array}{lll}
0 & 1 & 0 \\
0 & 0 & 0 \\
0 & 0 & 0
\end{array}\right],
\end{array}
$$

$r=3, n=\operatorname{deg} \operatorname{det}(A(s))=2$. The finite Jordan pairs of $A(s)$ is $(C, J)$ :

$$
C=\left[\begin{array}{cc}
0 & 0 \\
0 & -1 \\
1 & 1
\end{array}\right], \quad J=\left[\begin{array}{ll}
0 & 1 \\
0 & 0
\end{array}\right]
$$


The Smith-McMillan form of $A(s)$ at $s=\infty$ is

$$
S_{A(s)}^{\infty}(s)=\operatorname{diag}\left[s^{3}, s^{2}, 1 / s^{3}\right]
$$

so $A(s)$ has two poles at $s=\infty$ with orders $q_{1}=3$ and $q_{2}=2$ respectively and one zero at $s=\infty$ with order $\hat{q}_{3}=3$. The dual polynomial matrix to $A(s)$ is

$$
D_{A(s)}(w)=w^{q_{1}} A\left(\frac{1}{w}\right)=\left[\begin{array}{ccc}
w^{3} & 1 & 0 \\
0 & w^{3} & w^{2} \\
0 & 0 & w
\end{array}\right] \in R[w]^{3 \times 3}
$$

and

$$
\tilde{U}_{L}(w) D_{A(s)}(w) \tilde{U}_{R}(w)=S_{D_{A(s)}(w)}^{0}=w^{q_{1}} S_{A(s)}^{\infty}\left(\frac{1}{w}\right)=\left[\begin{array}{ccc}
1 & 0 & 0 \\
0 & w & 0 \\
0 & 0 & w^{6}
\end{array}\right]
$$

is the local Smith form of $D_{A(s)}(w)$ at $w=0$, where

$$
\tilde{U}_{L}(w)=\left[\begin{array}{ccc}
1 & 0 & 0 \\
0 & 0 & 1 \\
-w^{3} & 1 & -w
\end{array}\right], \quad \tilde{U}_{R}(w)=\left[\begin{array}{ccc}
0 & 0 & -1 \\
1 & 0 & w^{3} \\
0 & 1 & 0
\end{array}\right] .
$$

One easily obtains the generalized infinite Jordan pair $\left(C_{\infty}^{\prime}, J_{\infty}^{\prime}\right)$ (in the sense of Gohberg et al, 1982) of $A(s)$ as following

$$
\begin{gathered}
C_{\infty}^{\prime}=\left[\begin{array}{c|cccc|cc}
0 & -1 & 0 & 0 & 0 & 0 & 0 \\
0 & 0 & 0 & 0 & 1 & 0 & 0 \\
1 & 0 & 0 & 0 & 0 & 0 & 0
\end{array}\right], \\
J_{\infty}^{\prime}=\left[\begin{array}{c|cccccc}
0 & 0 & 0 & 0 & 0 & 0 & 0 \\
\hline 0 & 0 & 1 & 0 & 0 & 0 & 0 \\
0 & 0 & 0 & 1 & 0 & 0 & 0 \\
0 & 0 & 0 & 0 & 1 & 0 & 0 \\
0 & 0 & 0 & 0 & 0 & 1 & 0 \\
0 & 0 & 0 & 0 & 0 & 0 & 1 \\
0 & 0 & 0 & 0 & 0 & 0 & 0
\end{array}\right],
\end{gathered}
$$

and the infinite Jordan pair $\left(C_{\infty}, J_{\infty}\right)$ (in the sense of Vardulakis, 1991) of $A(s)$ as

$$
C_{\infty}=\left[\begin{array}{cccc}
-1 & 0 & 0 & 0 \\
0 & 0 & 0 & 1 \\
0 & 0 & 0 & 0
\end{array}\right], \quad J_{\infty}=\left[\begin{array}{cccc}
0 & 1 & 0 & 0 \\
0 & 0 & 1 & 0 \\
0 & 0 & 0 & 1 \\
0 & 0 & 0 & 0
\end{array}\right]
$$

It is easy to find $P$,

$$
P=\left[\begin{array}{lllllll}
0 & 0 & 0 & 0 & 1 & 0 & 0 \\
1 & 0 & 0 & 0 & 0 & 0 & 0 \\
0 & 1 & 0 & 0 & 0 & 0 & 0 \\
0 & 0 & 1 & 0 & 0 & 0 & 0 \\
0 & 0 & 0 & 1 & 0 & 0 & 0 \\
0 & 0 & 0 & 0 & 0 & 1 & 0 \\
0 & 0 & 0 & 0 & 0 & 0 & 1
\end{array}\right]
$$


such that

$$
\underbrace{\left[\begin{array}{ccccccc}
0 & -1 & 0 & 0 & 0 & 0 & 0 \\
0 & 0 & 0 & 0 & 1 & 0 & 0 \\
1 & 0 & 0 & 0 & 0 & 0 & 0
\end{array}\right]}_{C_{\infty}^{\prime}} \underbrace{\left[\begin{array}{lllllll}
0 & 0 & 0 & 0 & 1 & 0 & 0 \\
1 & 0 & 0 & 0 & 0 & 0 & 0 \\
0 & 1 & 0 & 0 & 0 & 0 & 0 \\
0 & 0 & 1 & 0 & 0 & 0 & 0 \\
0 & 0 & 0 & 1 & 0 & 0 & 0 \\
0 & 0 & 0 & 0 & 0 & 1 & 0 \\
0 & 0 & 0 & 0 & 0 & 0 & 1
\end{array}\right]}_{P}=\underbrace{\left[\begin{array}{cccc|ccc}
-1 & 0 & 0 & 0 & 0 & 0 & 0 \\
0 & 0 & 0 & 1 & 0 & 0 & 0 \\
0 & 0 & 0 & 0 & 0 \\
1 & 0 & 0
\end{array}\right]}_{C_{\infty}} \underbrace{\begin{array}{ll}
1 \\
0
\end{array}}_{C_{0}},
$$

$\underbrace{\left[\begin{array}{lllllll}0 & 1 & 0 & 0 & 0 & 0 & 0 \\ 0 & 0 & 1 & 0 & 0 & 0 & 0 \\ 0 & 0 & 0 & 1 & 0 & 0 & 0 \\ 0 & 0 & 0 & 0 & 1 & 0 & 0 \\ 1 & 0 & 0 & 0 & 0 & 0 & 0 \\ 0 & 0 & 0 & 0 & 0 & 1 & 0 \\ 0 & 0 & 0 & 0 & 0 & 0 & 1\end{array}\right]}_{P^{-1}} \underbrace{\left[\begin{array}{lllllll}0 & 0 & 0 & 0 & 0 & 0 & 0 \\ 0 & 0 & 1 & 0 & 0 & 0 & 0 \\ 0 & 0 & 0 & 1 & 0 & 0 & 0 \\ 0 & 0 & 0 & 0 & 1 & 0 & 0 \\ 0 & 0 & 0 & 0 & 0 & 1 & 0 \\ 0 & 0 & 0 & 0 & 0 & 0 & 1 \\ 0 & 0 & 0 & 0 & 0 & 0 & 0\end{array}\right]}_{J_{\infty}^{\prime}} \underbrace{\left[\begin{array}{lllllll}0 & 0 & 0 & 0 & 1 & 0 & 0 \\ 1 & 0 & 0 & 0 & 0 & 0 & 0 \\ 0 & 1 & 0 & 0 & 0 & 0 & 0 \\ 0 & 0 & 1 & 0 & 0 & 0 & 0 \\ 0 & 0 & 0 & 1 & 0 & 0 & 0 \\ 0 & 0 & 0 & 0 & 0 & 1 & 0 \\ 0 & 0 & 0 & 0 & 0 & 0 & 1\end{array}\right]}_{P}=$

$$
\left[\begin{array}{llll|lll}
0 & 1 & 0 & 0 & 0 & 0 & 0 \\
0 & 0 & 1 & 0 & 0 & 0 & 0 \\
0 & 0 & 0 & 1 & 0 & 0 & 0 \\
0 & 0 & 0 & 0 & 0 & 1 & 0 \\
\hline 0 & 0 & 0 & 0 & 0 & 0 & 0 \\
0 & 0 & 0 & 0 & 0 & 0 & 1 \\
0 & 0 & 0 & 0 & 0 & 0 & 0
\end{array}\right]:=\left[\begin{array}{c|c}
J_{\infty} & J^{\prime} \\
\hline 0 & J_{0}
\end{array}\right]
$$

According to Lemma 2, one has

$$
\begin{aligned}
V & =\left[A_{3} C J^{2},-\left(A_{0} C_{\infty}^{\prime}\left(J_{\infty}^{\prime}\right)^{2}+A_{1} C_{\infty}^{\prime} J_{\infty}^{\prime}+A_{2} C_{\infty}^{\prime}\right)\right] \\
= & {\left[\begin{array}{cc|ccccccc}
0 & 0 & 0 & 0 & 0 & 1 & 0 & 0 & 0 \\
0 & 0 & 0 & 0 & 0 & 0 & 0 & 0 & -1 \\
0 & 0 & -1 & 0 & 0 & 0 & 0 & 0 & 0
\end{array}\right] } \\
S= & {\left[\begin{array}{c|c|c|ccccc}
C & C_{\infty}^{\prime} J_{\infty}^{\prime} \\
C J & C_{\infty}^{\prime}
\end{array}\right] } \\
= & {\left[\begin{array}{cc|ccccccc}
0 & 0 & 0 & 0 & -1 & 0 & 0 & 0 & 0 \\
0 & -1 & 0 & 0 & 0 & 0 & 0 & 1 & 0 \\
1 & 1 & 0 & 0 & 0 & 0 & 0 & 0 & 0 \\
\hline 0 & 0 & 0 & -1 & 0 & 0 & 0 & 0 & 0 \\
0 & 0 & 0 & 0 & 0 & 0 & 1 & 0 & 0 \\
0 & 1 & 1 & 0 & 0 & 0 & 0 & 0 & 0
\end{array}\right] }
\end{aligned}
$$

SO

$$
\begin{aligned}
Z^{\prime} & =\left[\begin{array}{l}
Z_{1} \\
Z_{2}^{\prime}
\end{array}\right] \\
& =\left[\begin{array}{c|c}
I_{n} & 0 \\
\hline 0 & \left(J_{\infty}^{\prime}\right)^{q_{1}-1}
\end{array}\right]\left[\begin{array}{c}
S \\
V
\end{array}\right]^{-1}[0, \cdots, 0, I]^{T}
\end{aligned}
$$




$$
\begin{aligned}
& {\left.\left[\begin{array}{cc|ccccccc}
1 & 0 & 0 & 0 & 0 & 0 & 0 & 0 & 0 \\
0 & 1 & 0 & 0 & 0 & 0 & 0 & 0 & 0 \\
\hline 0 & 0 & 0 & 0 & 0 & 0 & 0 & 0 & 0 \\
0 & 0 & 0 & 0 & 0 & 1 & 0 & 0 & 0 \\
0 & 0 & 0 & 0 & 0 & 0 & 1 & 0 & 0 \\
0 & 0 & 0 & 0 & 0 & 0 & 0 & 1 & 0 \\
0 & 0 & 0 & 0 & 0 & 0 & 0 & 0 & 1 \\
0 & 0 & 0 & 0 & 0 & 0 & 0 & 0 & 0 \\
0 & 0 & 0 & 0 & 0 & 0 & 0 & 0 & 0
\end{array}\right]\left[\begin{array}{cc|ccccccc}
0 & 0 & 0 & 0 & -1 & 0 & 0 & 0 & 0 \\
0 & -1 & 0 & 0 & 0 & 0 & 0 & 1 & 0 \\
1 & 1 & 0 & 0 & 0 & 0 & 0 & 0 & 0 \\
0 & 0 & 0 & -1 & 0 & 0 & 0 & 0 & 0 \\
0 & 0 & 0 & 0 & 0 & 0 & 1 & 0 & 0 \\
0 & 1 & 1 & 0 & 0 & 0 & 0 & 0 & 0 \\
\hline 0 & 0 & 0 & 0 & 0 & 1 & 0 & 0 & 0 \\
0 & 0 & 0 & 0 & 0 & 0 & 0 & 0 & -1 \\
0 & 0 & -1 & 0 & 0 & 0 & 0 & 0 & 0
\end{array}\right] \begin{array}{ccc}
0 \\
0 & 0 & -1 \\
0 & 0 & 1 \\
0 & 0 & 0 \\
1 & 0 & 0 \\
0 & 0 & 0 \\
0 & 0 & 1 \\
0 & -1 & 0 \\
0 & 0 & 0 \\
0 & 0 & 0
\end{array}\right] . }
\end{aligned}
$$

We obtain

$$
Z_{1}=\left[\begin{array}{ccc}
0 & 0 & -1 \\
0 & 0 & 1
\end{array}\right], \quad Z_{2}^{\prime}=\left[\begin{array}{c}
Z_{21}^{\prime} \\
Z_{22}^{\prime}
\end{array}\right]=\left[\begin{array}{ccc}
0 & 0 & 0 \\
1 & 0 & 0 \\
0 & 0 & 0 \\
0 & 0 & 1 \\
\hline 0 & -1 & 0 \\
0 & 0 & 0 \\
0 & 0 & 0
\end{array}\right]
$$

and from

$$
\begin{aligned}
P^{-1} Z_{2}^{\prime}= & {\left[\begin{array}{lllllll}
0 & 1 & 0 & 0 & 0 & 0 & 0 \\
0 & 0 & 1 & 0 & 0 & 0 & 0 \\
0 & 0 & 0 & 1 & 0 & 0 & 0 \\
0 & 0 & 0 & 0 & 1 & 0 & 0 \\
1 & 0 & 0 & 0 & 0 & 0 & 0 \\
0 & 0 & 0 & 0 & 0 & 1 & 0 \\
0 & 0 & 0 & 0 & 0 & 0 & 1
\end{array}\right]\left[\begin{array}{ccc}
0 & 0 & 0 \\
1 & 0 & 0 \\
0 & 0 & 0 \\
0 & 0 & 1 \\
0 & -1 & 0 \\
0 & 0 & 0 \\
0 & 0 & 0
\end{array}\right] } \\
= & {\left[\begin{array}{ccc}
1 & 0 & 0 \\
0 & 0 & 0 \\
0 & 0 & 1 \\
0 & -1 & 0 \\
0 & 0 & 0 \\
0 & 0 & 0 \\
0 & 0 & 0
\end{array}\right], }
\end{aligned}
$$


we obtain

$$
Z_{2}=\left[\begin{array}{ccc}
1 & 0 & 0 \\
0 & 0 & 0 \\
0 & 0 & 1 \\
0 & -1 & 0
\end{array}\right]
$$

Hence, by Theorem 2 we have constructed a resolvent form for the regular polynomial matrix $\mathrm{A}(\mathrm{s})$ as following

$$
\begin{aligned}
A^{-1}(s) & =\left[C, C_{\infty}\right]\left[\begin{array}{c|c}
I_{n} s-J & 0 \\
\hline 0 & J_{\infty} s-I_{\mu}
\end{array}\right]^{-1}\left[\begin{array}{c}
Z_{1} \\
Z_{2}
\end{array}\right] \\
& =\left[\begin{array}{cc|cccc}
0 & 0 & -1 & 0 & 0 & 0 \\
0 & -1 & 0 & 0 & 0 & 1 \\
1 & 1 & 0 & 0 & 0 & 0
\end{array}\right]\left[\begin{array}{cc|cccc}
s & -1 & 0 & 0 & 0 & 0 \\
0 & s & 0 & 0 & 0 & 0 \\
\hline 0 & 0 & -1 & s & 0 & 0 \\
0 & 0 & 0 & -1 & s & 0 \\
0 & 0 & 0 & 0 & -1 & s \\
0 & 0 & 0 & 0 & 0 & -1
\end{array}\right]\left[\begin{array}{ccc}
0 & 0 & -1 \\
0 & 0 & 1 \\
\hline 1 & 0 & 0 \\
0 & 0 & 0 \\
0 & 0 & 1 \\
0 & -1 & 0
\end{array}\right] .
\end{aligned}
$$

Based on Algorithm 1, a procedure in the symbolic computational package Maple has been developed and has been implemented to carry out the above calculations. Note that in the above refined resolvent decomposition the matrices $C_{\infty}, J_{\infty}$ and $Z_{2}$ have the minimal dimensions of $3 \times 4,4 \times 4$ and $4 \times 3$. Compared to the resolvent decomposition of Gohberg et al (1982) given by

$$
A^{-1}(s)=\left[C, C_{\infty}^{\prime}\right]\left[\begin{array}{c|c}
I_{n} s-J & 0 \\
\hline 0 & J_{\infty}^{\prime} s-I_{\nu}
\end{array}\right]^{-1}\left[\begin{array}{c}
Z_{1} \\
Z_{2}^{\prime}
\end{array}\right],
$$

where $C_{\infty}^{\prime} \in \Re^{3 \times 7}, J_{\infty}^{\prime} \in \Re^{7 \times 7}$ and $Z_{2}^{\prime} \in \Re^{7 \times 3}$ are given by (14), (15) and (16) respectively, due to the fact that the redundant information has been deleted, the above refined resolvent decomposition is obviously in a much more concise form which will definitely bring some convenience when applied to the solution of the regular PMDs.

\section{Conclusion}

So far there have been two special resolvent decompositions proposed in the literature through which the solution of a PMD may be expressed. These are based on two different interpretations of the notion of infinite Jordan pair, the first being due to Gohberg et al (1982), and the second due to Vardulakis (1991). The resolvent decomposition proposed by Gohberg et al (1982) uses certain redundant system structure which results in overly large dimensions of the infinite Jordan pair, though it is relatively simple to calculate the infinite Jordan pair. On the other hand, the approach proposed by Vardulakis (1991) uses only the relevant system structure, without using redundant information, and the resulting infinite Jordan pair is of minimal dimension. It is however relatively more difficult to compute the required special realizations. 
In this paper, it is established that the redundant information contained in the infinite Jordan pair defined by Gohberg et al (1982) can be deleted through a certain transformation. Based on this, a natural connection between the infinite Jordan pairs defined by Gohberg et al (1982) and that of Vardulakis (1991) has been exploited. This facilitates a refinement of the resolvent decomposition. This resulting resolvent decomposition more precisely reflects the relevant system structure and thereby inherits the advantages of both the decompositions of Gohberg et al (1982) and Vardulakis (1991). In the proposed approach the matrices $Z, Z_{\infty}$ in (3) are formulated explicitly, which means this method is constructive. The main idea in this proposed approach is to calculate an elementary matrix $P$, which is very easy to obtain, to delete the redundant information, then to propose the refined resolvent decomposition. This elementary matrix has the effect of deleting the redundant information in two ways. First, it deletes the redundant information in those blocks in the infinite Jordan pair of Gohberg et al (1982) which corresponds to the infinite zeros and bring them into the correct sizes. Second, it deletes the whole blocks in the infinite Jordan pair of Gohberg et al (1982) which corresponds to the infinite poles and the whole blocks which are not dynamically important. This elementary matrix serves to transform the partitioned block matrix in $Z^{\prime}$ which corresponds to the redundant information into zero, the resulting refined resolvent decomposition is thus of minimal dimensions. Further by using this elementary matrix the mechanism of decoupling in the solution of Gohberg et al (1982) is explained clearly. This refined resolvent decomposition facilitates computation of the inverse matrix of $A(s)$ due to the fact that the dimensions of the matrices used are minimal. Once the refined resolvent decomposition is obtained, the generalized infinite Jordan pair and the elementary matrix $P$ are no longer needed in the calculation of the solution of the regular PMD. This thus presents another merit of this method which is algorithmically attractive when applied in actual computation.

Based on this refined resolvent decomposition, the complete solution of regular PMDs has then been investigated. This solution presents the zero input response and the zero state response precisely and takes into account the impulsive properties of the system. An algorithm, which has already been implemented in the symbolic computational package Maple, for the investigation of this refined resolvent decomposition is provided.

\section{Acknowledgment}

The authors would like to thank Dr M. Hou and the expert reviewers for suggestions and insightful comments

\section{References}

Fossard, A., 1972, Commande des Systemes Multidimensionnels, France: Dunod.

Gohberg, I., Langaster P. and Rodman I., 1982, Matrix Polynomials, Academic Press, New York.

Hayton, G. E., Pugh A. C. and Fretwell P., 1988, Infinite elementary divisors of a matrix polynomial and its implications, Int. J. Control, 47, pp. 53-64. 
Vardulakis, A. I. G., 1991, Linear Multivariable Control, Algebraic Analysis and Synthesis Methods, John Willey and Sons.

Verghese, G.C., Lévy B.C. and Kailath, T., 1981, A generalized state-space for singular system. IEEE Trans. on Automatic Control, AC-26, 811-831.

Pugh, A. C., 1976, The McMillan degree of a polynomial system matrix, Int. J. Control, vol. 24, No. 1, 129-135. 